\title{
Lentivirus-mediated silencing of CNTN1 enhances gefitinib sensitivity by reversing epithelial-mesenchymal transition in lung adenocarcinoma A549 cells
}

\author{
CHUN-SHENG HU ${ }^{1}$, JIU-HONG HUANG ${ }^{1}$, DONG-LIN YANG ${ }^{1}$, CHUAN XU $^{2}$, \\ ZHI-GANG XU ${ }^{1}$, HONG-BO TAN ${ }^{1}$ and ZHONG-ZHU CHEN ${ }^{1}$

\begin{abstract}
${ }^{1}$ College of Pharmacy, National and Local Joint Engineering Research Center of Targeted and Innovative Therapeutics, Chongqing Key Laboratory of Kinase Modulators as Innovative Medicine, Chongqing University of Arts and Sciences, Chongqing 402160; ${ }^{2}$ Department of Oncology, Sichuan Cancer Center, School of Medicine, University of Electronic Science and Technology of China, Chengdu, Sichuan 610047, P.R. China
\end{abstract}

Received May 16, 2020; Accepted March 4, 2021

DOI: $10.3892 / \mathrm{ol} .2021 .12694$

\begin{abstract}
Contactin-1 (CNTN1), a neuronal cell adhesion molecular, functions in nervous system development and has been associated with carcinogenesis and tumor progression. To investigate the role of CNTN1 in gefitinib resistance in lung adenocarcinoma, lentivirus-mediated short hairpin (sh)RNA was used to silence CNTN1 and its physiological function was analyzed in the A549 cell line. A cell cytotoxicity assay revealed that CNTN1 knockdown enhanced gefitinib sensitivity in the A549 cells. In addition, CNTN1 knockdown, together with gefitinib treatment, resulted in a significant inhibition of colony formation and migration, and promotion of apoptosis. Furthermore, CNTN1 knockdown also reversed the epithelial-mesenchymal transition (EMT) phenotype by increasing E-cadherin protein expression level, and decreasing $\mathrm{N}$-cadherin and vimentin protein expression levels. The PI3K/Akt signaling pathway was also association with the effects of CNTN1 on EMT progression and gefitinib resistance in the A549 cells. Collectively, knockdown of CNTN1 reversed the EMT phenotype and enhanced gefitinib sensitivity in the A549 cells by inhibiting the activation of the PI3K/Akt signaling pathway. These results suggested that CNTN1 may represent a potential therapeutic target
\end{abstract}

Correspondence to: Dr Hong-Bo Tan or Professor Zhong-Zhu Chen, College of Pharmacy, National and Local Joint Engineering Research Center of Targeted and Innovative Therapeutics, Chongqing Key Laboratory of Kinase Modulators as Innovative Medicine, Chongqing University of Arts and Sciences, 319 Honghe Avenue, Chongqing 402160, P.R. China

E-mail: 13167859296@163.com

E-mail: 18883138277@163.com

Key words: CNTN1, gefitinib, resistance, epithelial-mesenchymal transition, Akt for reserving EGFR-tyrosine kinase inhibitor resistance in non-small cell lung cancer.

\section{Introduction}

Lung cancer is the most common malignant tumor with $\sim 1.6$ million cases of lung cancer-associated mortality worldwide per year (1). The most common subtype is non-small cell lung cancer (NSCLC), which accounts for $~ 85 \%$ of all lung cancer cases (2). The therapeutic strategies include surgery, traditional chemotherapy, chemo- and radiotherapy, and molecular targets for NSCLC therapy. However, treatment failure in patients with NSCLC results from a lack of effective therapeutic strategies (2). Recent studies showed that patients receiving epidermal growth factor receptor-tyrosine kinase inhibitors (EGFR-TKIs), such as gefitinib or erlotinib, exhibited higher positive response rates and longer progression-free survival times compared with that in patients receiving traditional chemotherapy, in the majority of non-smoking patients with adenocarcinoma of Asian descent (3-5). However, most patients eventually experience tumor progression due to acquired resistance within $\sim 12$ months of gefitinib treatment (6). Therefore, EGFR-TKI resistance in patients with NSCLC is a critical problem associated with EGFR-TKI treatment.

The mechanism of resistance to EGFR-TKIs has been reported to include a secondary mutation (T790M), amplification of MET, a Ras mutation, loss of PTEN and a change in epithelial-to-mesenchymal transition (EMT) (7); however, the molecular mechanism of primary resistance is still poorly understood. Increasing evidence has suggested that EMT plays a vital role in EGFR-TKIs resistance. The rate of EMT accounted for $14 \%$ of cases of acquired EGFR-TKIs resistance in NSCLC (8). In addition, reversal of EMT improved gefitinib sensitivity in HCC827 resistant cells or lung tumors (9-11). However, a comprehensive understanding of the EMT regulatory mechanism contributing to EGFR-TKIs resistance remains necessary.

Contactin-1 (CNTN1), a neuronal cell adhesion molecular, has been reported to be associated with carcinogenesis and 
tumor progression. CNTN1 has alternative functions causing invasion and metastasis of various types of cancer, including prostate cancer (12), gastric cancer (13), hepatocellular carcinoma (14), breast cancer (15), thyroid cancer (16) and lung adenocarcinoma (LUAD) (17). Furthermore, overexpression of CNTN1 has been associated with poor prognosis in patients with oral squamous cell carcinoma (18). Knockdown of CNTN1 expression decreased invasion and metastasis, and inhibited tumor initiation in gastric and prostate cancers $(13,19)$. However, the function of CNTN1 in EGFR-TKI resistance remains unclear. In the current study, the role of CNTN1 in gefitinib resistance was evaluated and the molecular mechanism associated with gefitinib resistance induced by CNTN1 was investigated.

\section{Materials and methods}

Public databases. Gene Expression Omnibus (GEO; https://www.ncbi.nlm.nih.gov/gds) was used for the analysis of CNTN1 mRNA expression levels between gefitinib-sensitive and -insensitive NSCLC. The gene expression datasets (GSE4342 and GSE38302) were downloaded from the GEO database $(20,21)$. The differential expression of genes between gefitinib-sensitive cells (HCC827 and Calu3) and intrinsically resistant cells (A549, H460 and H1975) in GSE4342, and between acquired gefitinib resistant cells (PC9GR) and parental cells (PC9) in GSE38302 was determined using GEO2R (http://www.ncbi.nlm.nih.gov/geo/geo2r). The Kaplan-Meier plot was used to determine overall survival (OS) time using Kaplan-Meier Plotter database (https://kmplot.com) (22) and a $\log$ rank test. $\mathrm{P}<0.05$ was considered to indicate a statistically significant difference. The total number of patients with LUAD was 719, which were divided into the high and low expression level groups using an auto select best cutoff, including 536 $(74.5 \%)$ in the low CNTN1 expression group and $183(25.5 \%)$ in the high CNTN1 expression group.

Cell line and plasmids. The NSCLC A549 cell line was purchased from the American Type Culture Collection, and was cultured in high-glucose DMEM (Hyclone; Cytiva), supplemented with 10\% FBS (Natocor-Industria Biológica) and 1\% penicillin-streptomycin (Sigma-Aldrich; Merck KGaA). The cell line was thawed every month. Short hairpin (sh)RNA-CNTN1 and control shRNA [Luciferase shRNA (shLuc)] was purchased from Sigma-Aldrich (cat. no. SHC007; Merck KGaA). shRNA-CNTN1 was ligated into a lentiviral plasmid (Takara Bio Inc.) and the shRNA had the following sequences: shCNTN1-1, 5'-CCGGGCCGTGGTTCA GACAATCATACTCGAGTATGATTGTCTGAACCACGG CTTTTTG-3'; and shCNTN1-2, 5'-CCGGCCAAGGATCAT CAGTTCAGTACTCGAGTACTGAACTGATGATCCTTG GTTTTTG-3'.

Virus packaging and transfection. Briefly, the 293T cells were purchased from the American Type Culture Collection and were cultured in high-glucose DMEM, supplemented with $10 \%$ FBS. The cells were cultured to $50-60 \%$ confluency and co-transfected with either $10 \mu \mathrm{g}$ control (shLuc) or shRNA-CNTN1, with a packaging plasmid [7.5 $\mu \mathrm{g}$ psPAX2 (Invitrogen; Thermo Fisher Scientific Inc.)] and an envelope plasmid [2.5 $\mu \mathrm{g}$ pMD2.G (Invitrogen; Thermo Fisher Scientific
Inc.)] using $40 \mu \mathrm{g}$ PEI reagent (Sigma-Aldrich; Merck KGaA). The lentiviral particles-containing medium was harvested after transfection for $48 \mathrm{~h}$ at $37^{\circ} \mathrm{C}$ and filtered using a $0.22-\mu \mathrm{m}$ filter. The A549 cells were seeded into 6-well culture plates, at a density of $5 \times 10^{5}$ cells per well. When the confluency of the cells was $50-60 \%$, the culture medium was replaced with the $2 \mathrm{ml}$ lentiviral particles mixed with $4 \mu \mathrm{l}$ polybrene (Sigma-Aldrich; Merck KGaA) for $24 \mathrm{~h}$. Next, the cells were selected for stable integration with $4 \mu \mathrm{g}$ puromycin dihydrochloride for 2 weeks (2 $\mu \mathrm{g} / \mathrm{ml}$; Sigma-Aldrich; Merck KGaA). After this process, the stable cells were used for all further experiments and untransfected cells were used as a negative control.

In vitro drug sensitivity assay. For the MTT assay, the cells were seeded into a 96-well plate (5,000 cells/well) and incubated at $37^{\circ} \mathrm{C}$ in a humidified incubator with $5 \% \mathrm{CO}_{2}$. After being cultured for $24 \mathrm{~h}$, the cells were treated with increasing concentrations of gefitinib $(0,1,2,5,12.5,25$ and $50 \mu \mathrm{M})$ for $48 \mathrm{~h}$. The culture medium was replaced with fresh medium containing MTT solution $(0.5 \mathrm{mg} / \mathrm{ml}$; Sigma-Aldrich; Merck KGaA). After an additional 4-h incubation, the blue MTT-formazan product was dissolved in $200 \mu \mathrm{l}$ DMSO for $30 \mathrm{~min}$. The absorbance of the formazan solution was read spectrophotometrically at $570 \mathrm{~nm}$. The $50 \%$ inhibitory concentration $\left(\mathrm{IC}_{50}\right)$ was calculated using GraphPad Prism v7.0 software (GraphPad Software, Inc.).

Migration assays. A wound healing assay was performed to evaluate the migration ability of the A549 cells. Briefly, after the cells had been seeded in a 12 -well plate $\left(2 \times 10^{5}\right.$ cells per well) and cultured overnight, the $100 \%$ confluent cell monolayer was sequentially scratched in a straight line with a 200- $\mu 1$ pipette tip, washed with sterile PBS twice and cultured at $37^{\circ} \mathrm{C}$ in fresh FBS-free medium for $24 \mathrm{~h}$. Images of the migrated cells were captured at 0 and $24 \mathrm{~h}$ under a light microscope (magnification, x10) (Zeiss AG). The cell migration ability in each group was calculated using ImageJ software [version 1.52a; National Institutes of Health (NIH)].

The migration ability assay was performed according to the manufacturer's procedures (Corning Inc.). Briefly, a total of $5 \times 10^{4}$ A549 cells in $0.5 \mathrm{ml}$ serum-free DMEM were seeded onto a Transwell plate, with an $8-\mu \mathrm{m}$ pore size polycarbonate membrane. Next, $0.5 \mathrm{ml}$ DMEM with $10 \%$ FBS was placed in the lower chamber. After $24 \mathrm{~h}$, the cells that had migrated to the lower surface of the membranes were fixed with $4 \%$ paraformaldehyde for $30 \mathrm{~min}$ at room temperature and stained with crystal violet $(0.5 \%$; cat. no. C0775; Sigma-Aldrich; Merck $\mathrm{KGaA}$ ) for $2 \mathrm{~h}$ at room temperature. The number of cells that had migrated to the lower side of the membrane were counted using ImageJ software (version $1.52 \mathrm{a}$; NIH).

Colony formation. A549-shLuc and A549-shCNTN1-1 cells were seeded in a 6 -well plate $(1,000$ cells/well) and treated with DMSO or $12.5 \mu \mathrm{M}$ gefitinib for 2 weeks. The cells were subsequently stained with $0.5 \%$ crystal violet for $2 \mathrm{~h}$ at room temperature. Quantification was performed using ImageJ software (version 1.52a; NIH).

Cell cycle and apoptosis assay. For the cell cycle analysis, 2x105 A549-shLuc and A549-shCNTN1-1 cells were seeded 
in a 6-well plate. The cells were subsequently harvested and washed with PBS, then fixed with $70 \%$ ethanol for $30 \mathrm{~min}$ at room temperature. The fixed cells were then stained with fresh propidium iodide (PI) containing RNase A for $30 \mathrm{~min}$ at $37^{\circ} \mathrm{C}$. Finally, a total of $1 \times 10^{4}$ cells were analyzed from each sample using a BD Accuri C6 flow cytometer (BD Biosciences).

For apoptosis analysis, the transfected cells were seeded in a 6-well plate $\left(2 \times 10^{5}\right.$ cells/well) and treated with $12.5 \mu \mathrm{M}$ gefitinib. After incubation for $48 \mathrm{~h}$, the cells were digested with trypsin, washed with cold PBS twice, resuspended in $500 \mu 1$ binding buffer, then incubated with $5 \mu$ l FITC-Annexin V and $5 \mu \mathrm{l}$ PI for $15 \mathrm{~min}$ at room temperature, in the dark. Flow cytometry was performed using a BD Accuri C6 flow cytometer (BD Biosciences) following the manufacturer's instructions, to detect cell apoptosis by determining the relative amount of Annexin V-FITC-positive, PI-negative cells or FITC-Annexin V-positive and PI-positive cells. The cell cycle and apoptosis data analysis was performed using BD Accuri C6 Software v.1.0.264.21 (BD Biosciences).

Western blot analysis. Total protein from A549-shLuc and A549-shCNTN1-1 cells was extracted using RIPA lysis buffer (Beyotime Institute of Biotechnology) with a protease cocktail inhibitor (Roche). For detecting Akt phosphorylation (Thr308 and Ser473), the cells were harvested within 30 min after gefitinib or DMSO treatment to monitor the rapid Akt pathway activation. Protein concentration were measured using a BCA protein assay kit (Beyotime Institute of Biotechnology). Total protein $(20 \mu \mathrm{g} /$ lane $)$ was loaded and separated by $10 \%$ SDS-PAGE and transferred to $0.45-\mu \mathrm{m}$ PVDF membranes (EMD Millipore). Membranes were blocked with 5\% skimmed milk for $2 \mathrm{~h}$ at room temperature. The membranes were then incubated with the following specific monoclonal or polyclonal primary antibodies overnight at $4^{\circ} \mathrm{C}$ : Anti-CNTN1 (1:200; cat. no. sc-136133; Santa Cruz Biotechnology, Inc.), anti-Akt Ser473 phosphorylation (1:1,000; cat. no. 4060; Cell Signaling Technology, Inc.), anti-Akt Thr308 phosphorylation (1:1,000; cat. no. 9275; Cell Signaling Technology, Inc.), anti-Akt (1:1,000; cat. no. 9275; Cell Signaling Technology, Inc.), anti-E-cadherin (1:200; cat. no. sc-8426; Santa Cruz Biotechnology, Inc.), anti-N-cadherin (1:200; cat. no. sc-7939; Santa Cruz Biotechnology, Inc.), anti-vimentin (1:200; cat. no. sc-6260; Santa Cruz Biotechnology, Inc.) and anti-Actin (1:5,000; cat. no. A5441; Sigma-Aldrich; Merck $\mathrm{KGaA}$ ). Subsequently, the membranes were incubated with the corresponding horseradish peroxidase-conjugated anti-mouse IgG (1:10,000; cat. no. 7076; Cell Signaling Technology, Inc.) or anti-rabbit IgG antibodies (1:10,000; cat. no. 7074; Cell Signaling Technology, Inc.) for $1 \mathrm{~h}$ at room temperature. Signal detection was performed using an enhanced chemiluminescence kit (GE Healthcare) and photographed using an imaging system (Epson). Finally, data were analyzed using ImageJ software v.1.52a (National Institutes of Health).

Immunofluorescence staining. The cells were seeded on 8 -well cell culture slides, fixed with $4 \%$ paraformaldehyde for $30 \mathrm{~min}$ at room temperature and blocked with $3 \%$ BSA (Beyotime Institute of Biotechnology) in PBS for $2 \mathrm{~h}$ at room temperature. An anti- $\alpha$-tubulin (cat. no. ab24610; Abcam) antibody was added to the slides at a dilution of 1:200 and incubated overnight at $4^{\circ} \mathrm{C}$. The samples were then treated with an Alexa Fluor-conjugated anti-mouse antibody (1:1,000; cat. no. R37115; Thermo Fisher Scientific, Inc.) for $1 \mathrm{~h}$ protected from light at room temperature. The nuclei were stained with DAPI (Thermo Fisher Scientific, Inc.) for $15 \mathrm{~min}$ at room temperature. Images were captured using a Zeiss fluorescence microscope (magnification, x20) (Zeiss AG).

Statistical analysis. All the data were obtained from three independent experiments and presented as the mean \pm standard deviation, with between-group differences analyzed by multiple t-tests followed by the Holm-Sidak correction test. $\mathrm{P}<0.05$ was considered to indicate a statistically significant difference. All statistical analyses were performed using GraphPad Prism v7.0 (GraphPad Software, Inc.).

\section{Results}

CNTN1 is abnormally upregulated in gefitinib-insensitive and-resistant NSCLC. To investigate the role of CNTN1 in the gefitinib resistance of NSCLC, the mRNA expression level of CNTN1 in gefitinib-sensitive and -insensitive NSCLC was analyzed using data from the GEO database. In the GSE4342 dataset, the mRNA expression level of CNTN1 in gefitinib-insensitive lung cancer cells was significantly higher compared with that in gefitinib-sensitive lung cancer cells $(\mathrm{P}<0.05$; Fig. 1A). In addition, the mRNA expression level of CNTN1 in gefitinib-acquired resistant cells was significantly higher compared with that in the parental cells according to the GSE38302 data $(\mathrm{P}<0.05$; Fig. 1B). Furthermore, the prognostic value of CNTN1 was analyzed using the Kaplan-Meier plotter database. The results showed that patients with LUAD and high mRNA expression levels of CNTN1 showed reduced overall survival times (Fig. 1C), which indicated that CNTN1 was upregulated in gefitinib-resistant and -insensitive NSCLC, and was associated with worse prognosis. Collectively, the results from publicly available data suggested that CNTN1 might play an important role in EGFR-TKI resistance.

CNTN1 knockdown inhibits the migration but not the proliferation ability of A549 cells. To further understand the function of CNTN1 in cell migration, CNTN1 was silenced in the high-metastatic LUAD A549 cell line using CNTN1-targeted shRNA. The western blot analysis showed that shCNTN1-1 and shCNTN1-2 markedly inhibited CNTN1 protein expression level (Fig. 2A). shCNTN1-1 was selected for subsequent experimentation due shCNTN1-1 and shCNTN1-2 having comparable effects. Transwell analysis confirmed that CNTN1 knockdown significantly decreased the migration ability of the A549 cells compared with untransfected A549 cells or A549 cells transfected with shLuc (Fig. 2B). To further understand the role of CNTN1 in the arrangement of the cytoskeleton and in adhesion structures which can affect cell motility, the A549-shLuc and A549-shCNTN1-1 cells were stained with $\alpha$-tubulin and characterized using a fluorescent microscope. The A549-shLuc cells displayed relaxing-formed $\alpha$-tubulin-containing microtubules within the cytoplasm and below the plasma membrane. However, the A549-shCNTN1-1 cells displayed firm-formed microtubules (Fig. 2C). Accordingly, these findings strongly suggested that CNTN1 could regulate the migration ability of LUAD cells. 

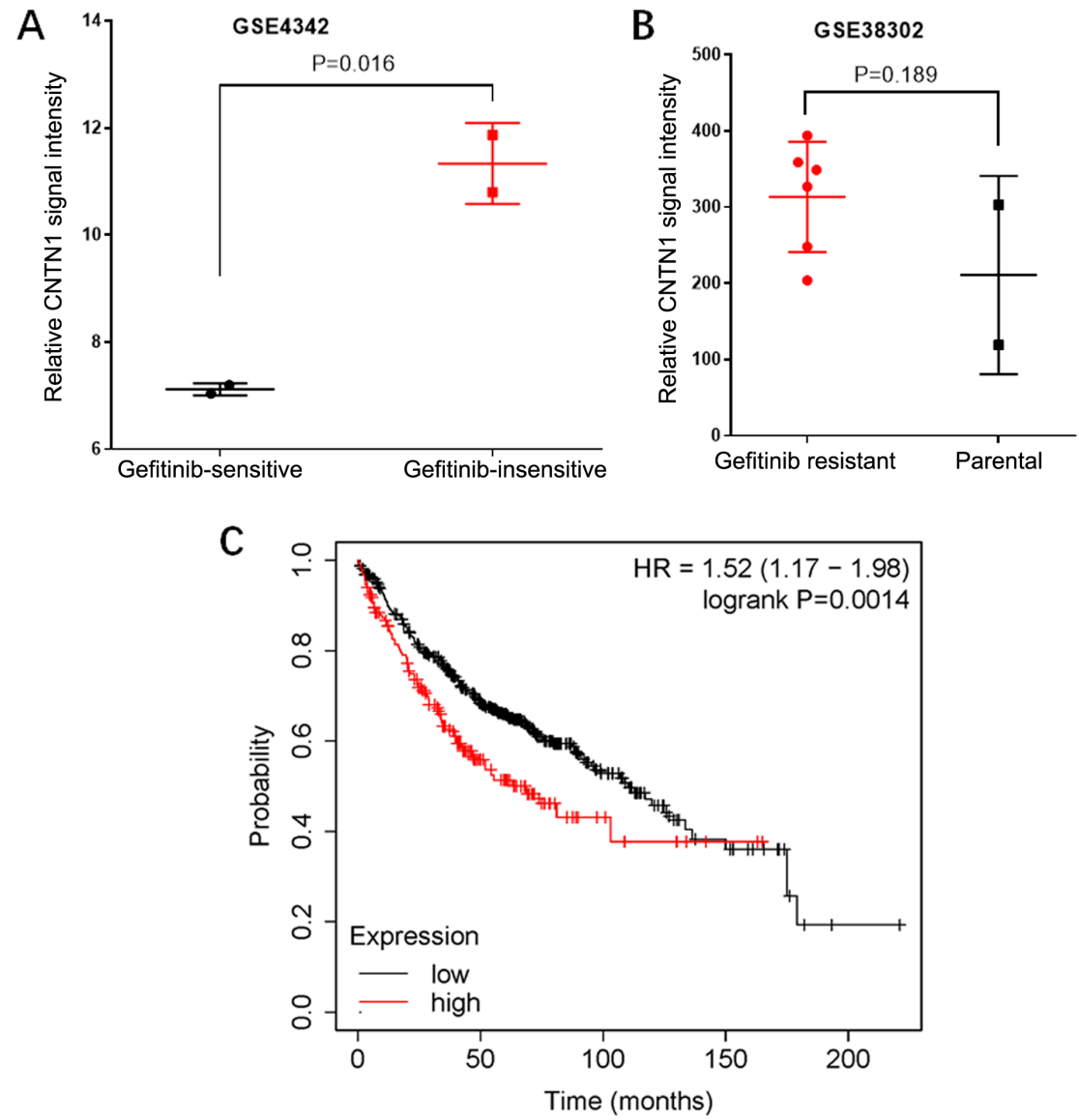

Figure 1. CNTN1 expression is upregulated in gefitinib-insensitive and -resistant non-small cell lung cancer cells from public databases. (A) GSE4342 dataset from the GEO database showed that the relative CNTN1 mRNA expression level in two gefitinib-insensitive cell lines was significantly higher compared with that in gefitinib-sensitive cell lines. (B) GSE38302 dataset showed that the CNTN1 mRNA expression level in six gefitinib-resistant lung cancer cell lines was higher compared with that in the corresponding parental cell lines. (C) The overall survival of patients with lung adenocarcinoma and high (n=183) or low $(n=536)$ mRNA expression levels of CNTN1 was plotted using the Kaplan-Meier method. HR, hazard ratio.

Next, the proliferation role of CNTN1 in the A549 cells transfected with shLuc or shCNTN1-1 was evaluated. MTT analyses showed that knockdown of CNTN1 was not associated with cell proliferation of A549 cells (Fig. 2D). Analysis of cell cycle distribution using flow cytometry showed that CNTN1 knockdown had no significant effect on the cell cycle in the A549 cells (Fig. 2E).

Knockdown of CNTN1 enhances gefitinib sensitivity in the A549 cells. To further understand the association between CNTN1 expression level and gefitinib sensitivity, the $\mathrm{IC}_{50}$ of the A549 cells transfected with shLuc or shCNTN1-1 was determined. As shown in Fig. 3A, the $\mathrm{IC}_{50}$ values of gefitinib in the A549-shCNTN1-1 and A549-shLuc cells were 14.45 and $28.33 \mu \mathrm{M}$, respectively, confirming that CNTN1 mRNA expression level could induce gefitinib resistance in the A549 cells.

In addition, the colony formation assay showed that CNTN1 knockdown of A549 treated with DMSO had no impact on colony formation capacity compared with the shLuc group treated with DMSO. However, the A549 cells transfected with shCNTN1-1 or shLuc were treated with $12.5 \mu \mathrm{M}$ gefitinib for 2 weeks and the numbers of colonies derived from the CNTN1-knockdown cells were significantly decreased compared with that in cells transfected with shLuc
( $\mathrm{P}<0.05$; Fig. 3B). These results indicated that knockdown of CNTN1 enhanced gefitinib sensitivity in the A549 cells.

CNTN1 knockdown enhances the effect of gefitinib on the migration of the A549 cells. As CNTN1 could promote cell migration in previous studies $(15,23)$, it was hypothesized that knockdown of CNTN1 could enhance the effect of gefitinib on inhibition of the cell migration abilities of A549 cells. Transwell and wound healing assays were used to investigate the role of CNTN1 and gefitinib in combination on A549 cell migration. The number of migrated cells was significantly decreased in both knockdown of CNTN1 and gefitinib-treated group compared with that in the shLuc group treated with gefitinib (Fig. 4A). The results were also confirmed by the wound healing assay, in which the wound closure rate was significantly inhibited in A549 cells with knockdown of CNTN1 and gefitinib combination compared with that in cells treated with shLuc and gefitinib combination (Fig. 4C). Furthermore, western blot analysis revealed that the protein expression level of the epithelial marker, E-cadherin was increased, whereas the protein expression level of the mesenchymal markers (N-cadherin and vimentin) was notably decreased in the CNTN1-knockdown A549 cells (Fig. 4B). Collectively, these findings suggested that the EMT process was associated with malignant behavior in A549 cells via CNTN1. 


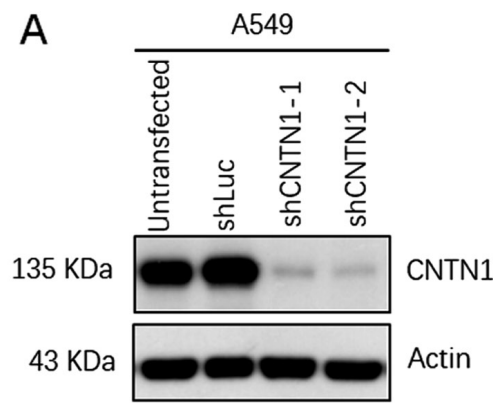

B
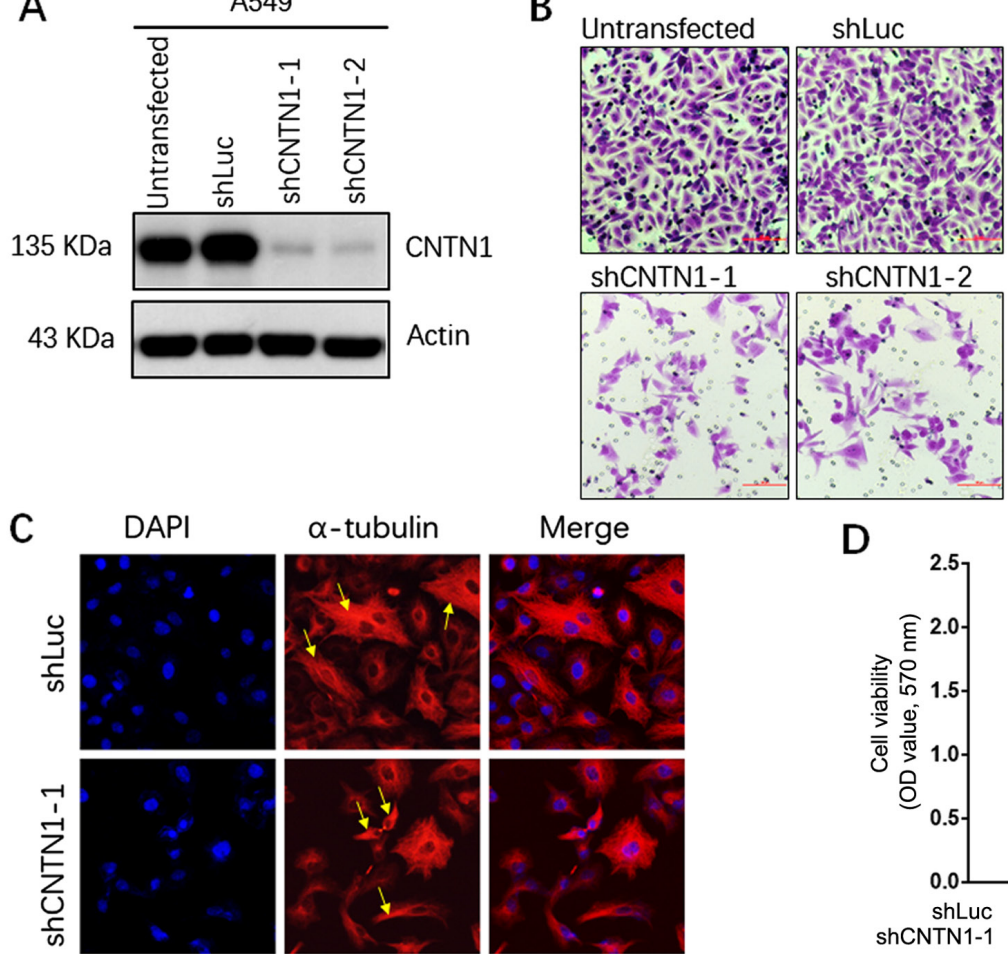

$\alpha$-tubulin

Merge
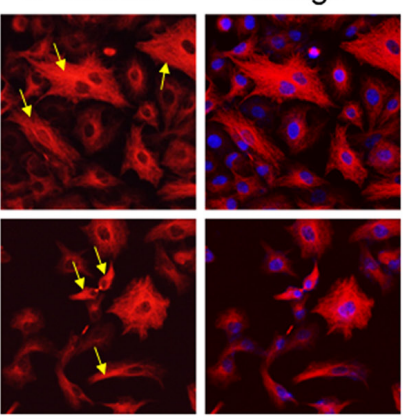

D

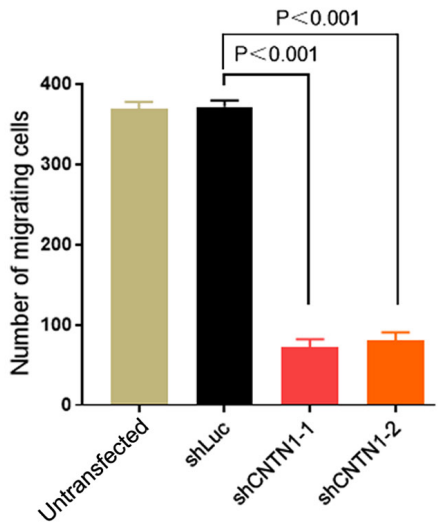

shLuc

\section{E}

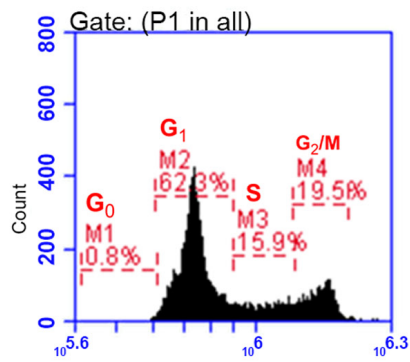

FL2-A

shCNTN1-1

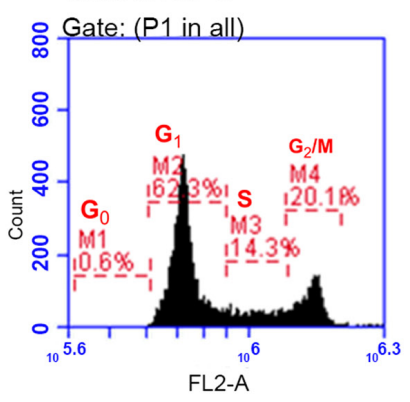

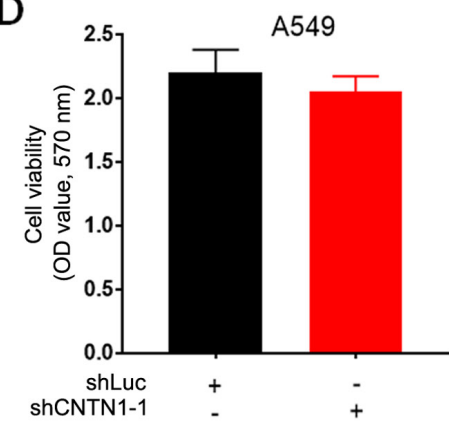

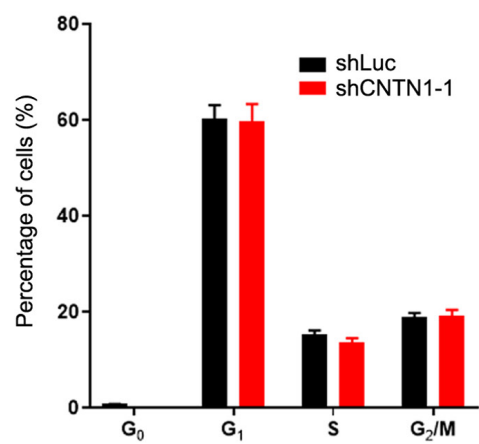

Figure 2. CNTN1 affects cell migration, cytoskeleton and cell proliferation in vitro. (A) The protein expression level of CNTN1 in the A549 cells stably expressing shLuc or CNTN1-targeted shRNAs was determined using western blot analysis. (B) Compared with that in the parental A549 or shLuc transfected A549 cells, the migration of both CNTN1-shRNA A549 cells was inhibited. (C) Presence of relaxing-formed $\alpha$-tubulin-containing microtubules in the A549-shLuc cells, but not in theA549-shCNTN1-1 cells. The cells were fixed and stained with DAPI to visualize the nuclei (blue) and with an anti- $\alpha$-tubulin antibody to detect the microtubules (red). Yellow arrows represent the changes of cytoskeleton. The A549-shLuc cells displayed relaxing-formed microtubules and the A549-shCNTN1-1 cells displayed firm-formed microtubules. (D) Cell proliferation of the A549 cells transfected with shCNTN1-1 or shLuc was determined at $570 \mathrm{~nm}$ following MTT assay. (E) Stably transfected cells (A549-shCNTN1-1 and A549-shLuc) were stained with PI and analyzed using flow cytometry for cell cycle analysis. The cell cycle distribution was not affected after silencing CNTN1 in the A549 cells. OD, optical density; sh, short hairpin; shLuc, control shRNA.

CNTN1 knockdown induces A549 cell apoptosis in the presence of gefitinib. As shown in Fig. 3A, MTT assay showed that knockdown of CNTN1 significantly improved gefitinib sensitivity in the A549 cells. Therefore, to confirm that CNTN1 knockdown inhibited the proliferation of the A549 cells in the presence of gefitinib, cell apoptosis was analyzed using Annexin V-FITC and PI double staining, followed by flow cytometry. The apoptosis rate of A549-shLuc/DMSO, A549-shLuc/gefitinib, A549-shCNTN1-1/DMSO and A549-shCNTN1-1/gefitinib cells was $5.29 \pm 1.22,3.94 \pm 2.28$, $4.32 \pm 1.82$ and $22.47 \pm 3.03 \%$, respectively. The results showed that cell apoptosis increased by $~ 18 \%$ in A549 cells transfected with shCNTN1-1 compared with that of cells transfected with shLuc in the presence of $12.5 \mu \mathrm{M}$ gefitinib (Fig. 5), indicating that cell apoptosis was induced by CNTN1 knockdown.
CNTN1 knockdown reverses gefitinib resistance by inhibiting the PI3K/Akt signaling pathway. The PI3K/Akt pathway is not only a vital signaling pathway in the regulation of cell survival and growth, but it is also an efficient target to promote drug sensitivity in various types of cancer cells (24). To further investigate the mechanism underlying CNTN1-mediated gefitinib resistance and the EMT phenotype, the expression level of proteins in the PI3K/Akt signaling pathway was analyzed. As shown in Fig. 6, western blot analysis confirmed that knockdown of CNTN1 in the A549 cells did not affect the protein expression levels of total Akt or GSK-3 $\beta$. However, there was a greater decrease in the protein expression level of phosphorylated (p) Akt (T308 and S473) and p-GSK-3 $\beta$ in the CNTN1-knockdown A549 cells treated with $12.5 \mu \mathrm{M}$ gefitinib for 30 min compared with A549 transfected with shLuc and treated with $12.5 \mu \mathrm{M}$ gefitinib for $30 \mathrm{~min}$ (Fig. 6). Considering these results, it was 

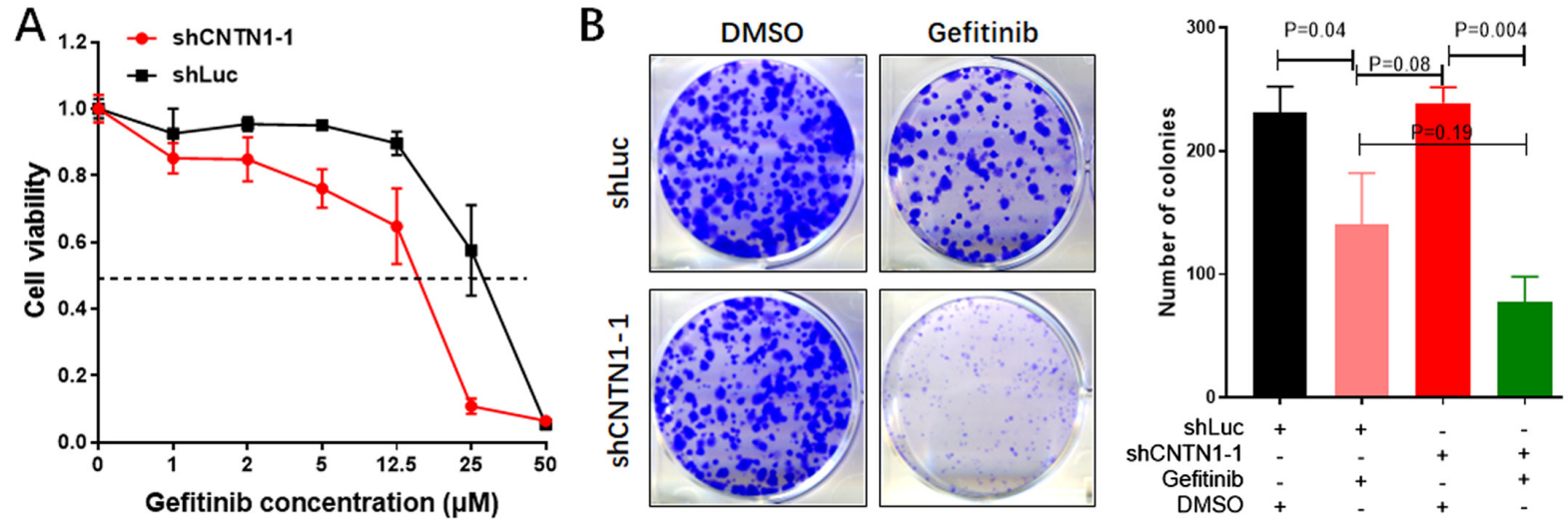

Figure 3. Knockdown of CNTN1 inhibits the viability and colony formation of the A549 cells in the presence of gefitinib. (A) The sensitivity of the A549 cells to gefitinib following transfection with shCNTN1-1 or shLuc was examined using a MTT assay. Stable knockdown of CNTN1 expression in the A549 cells significantly increased their sensitivity to gefitinib. (B) A549-shCNTN1-1 and A549-shLuc cells were treated with or without gefitinib and colony formation was determined 2 weeks later. There was a decrease in the colony formation ability of the A549-shCNTN1-1 cells treated with gefitinib compared with that in the A549-shLuc cells treated with gefitinib. DMSO, dimethyl sulfoxide; sh, short hairpin; shLuc, control shRNA.
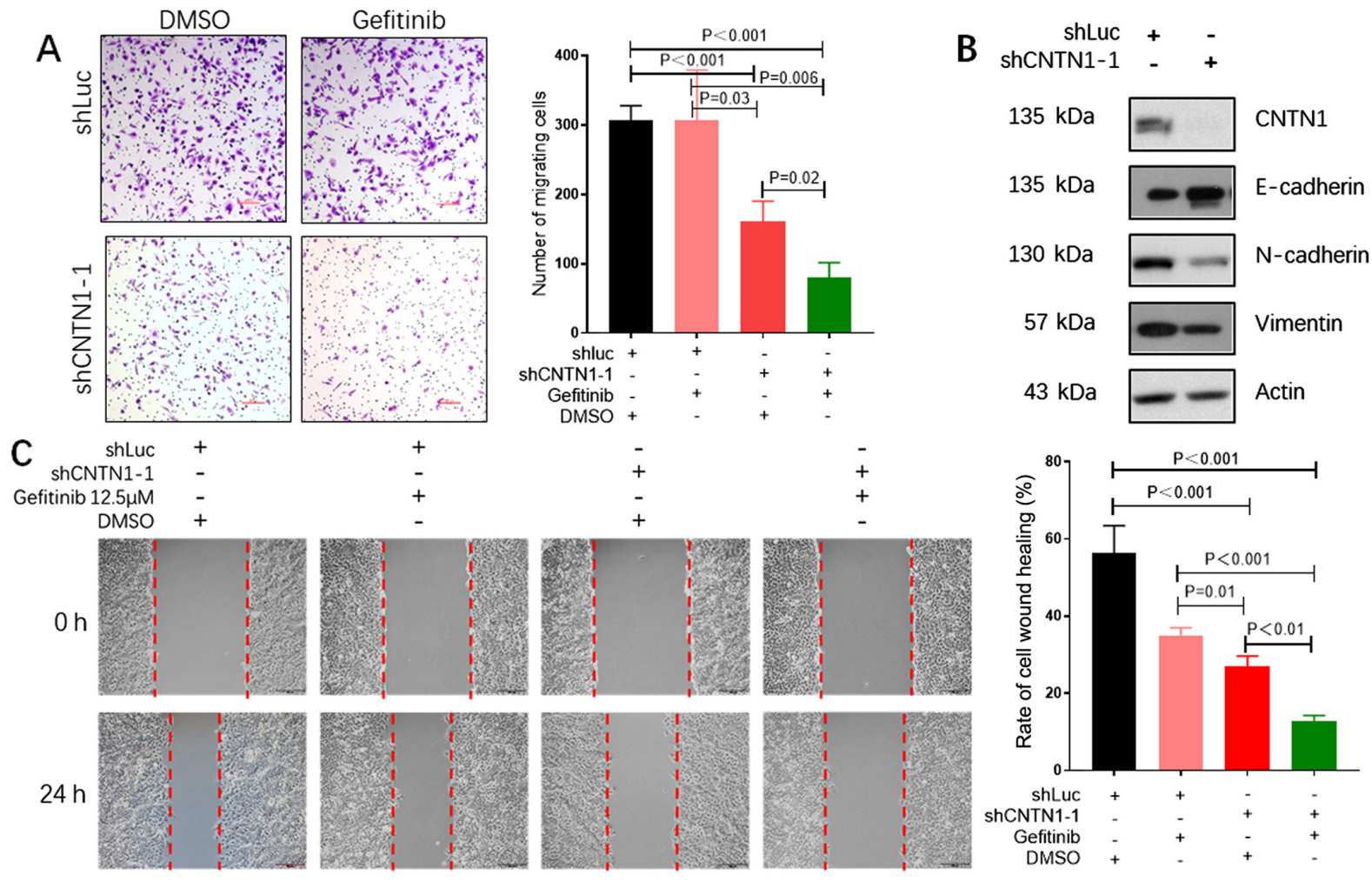

Figure 4. CNTN1 knockdown together with gefitinib treatment reduces the migration of the A549 cells via EMT. (A) Cell migration assay of the A549 cells transfected with shLuc or shCNTN1-1 and with or without gefitinib treatment was performed using a Transwell assay. The number of A549-shCNTN1-1 cells treated with gefitinib was significantly decreased compared with that in the A549-shLuc cell treated with gefitinib. (B) EMT markers were analyzed using western blotting. E-cadherin protein expression level was upregulated, while $\mathrm{N}$-cadherin and vimentin protein expression levels were downregulated in the A549 cells transfected with shCNTN1-1. (C) Cell migration ability was also assessed using a wound healing assay in the A549 cells transfected with shLuc or shCNTN1-1 and with or without gefitinib treatment. The combination of CNTN1 knockdown and gefitinib treatment significantly reduced the wound healing rate. EMT, epithelial-mesenchymal transition; sh, short hairpin; shLuc, control shRNA.

concluded that the regulatory effects of CNTN1 on gefitinib sensitivity and the EMT phenotype could be partially attributed to the PI3K/Akt/GSK-3 $\beta$ signaling pathway.

\section{Discussion}

Gefitinib, an EGFR-TKI, inhibits the intracellular tyrosine kinase domain of EGFR by competitively binding with ATP; thus, leading to the apoptosis of LUAD cells, and has been used as a first-line drug for NSCLC (25). However, the majority of patients develop acquired resistance to gefitinib within 12 months, which is still a main obstacle for the successful treatment of NSCLC (26). Further investigation of the potential mechanisms of gefitinib resistance may provide potential therapeutic strategies for addressing this challenge $(7,27,28)$. Several studies have reported that CNTN1 not only facilitated 

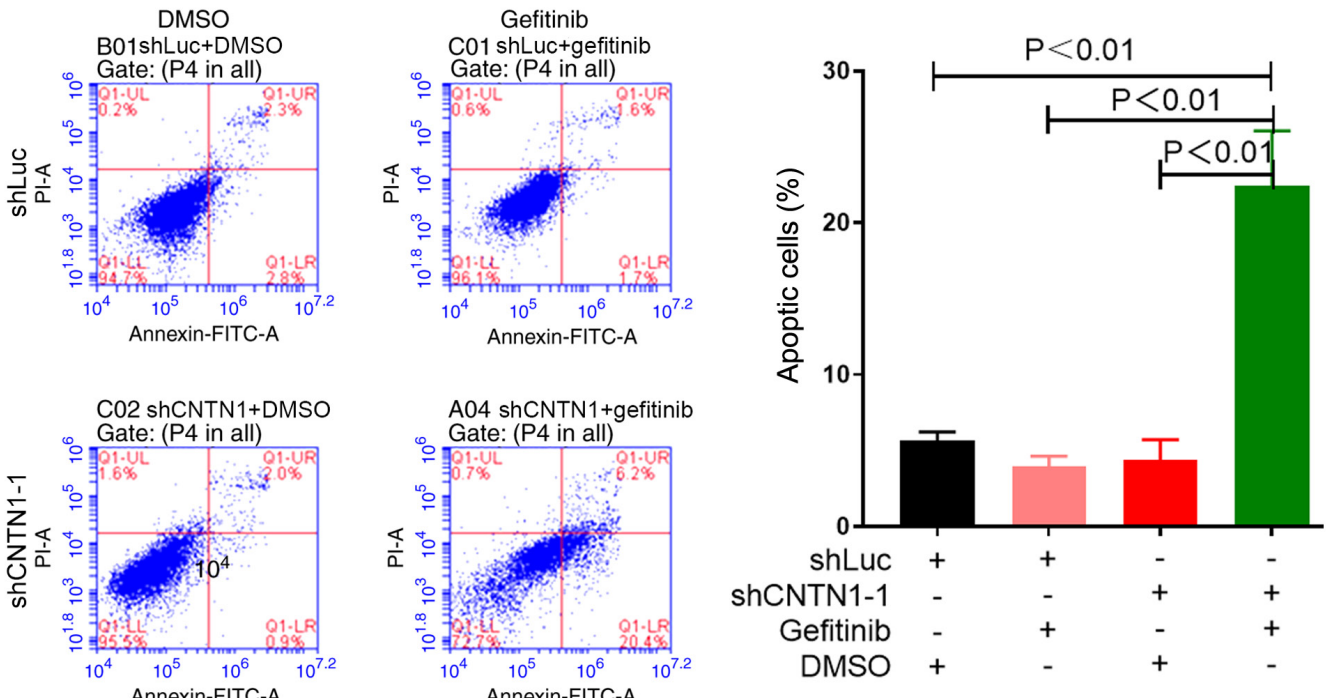

Figure 5. CNTN1 knockdown increases the rate of A549 cell apoptosis in the presence of gefitinib. The rate of apoptosis was evaluated following treatment of the A549-shCNTN1-1 and A549-shLuc cells with DMSO or 12.5 $\mu \mathrm{M}$ gefitinib for $48 \mathrm{~h}$. DMSO, dimethyl sulfoxide; sh, short hairpin; shLuc, control shRNA.
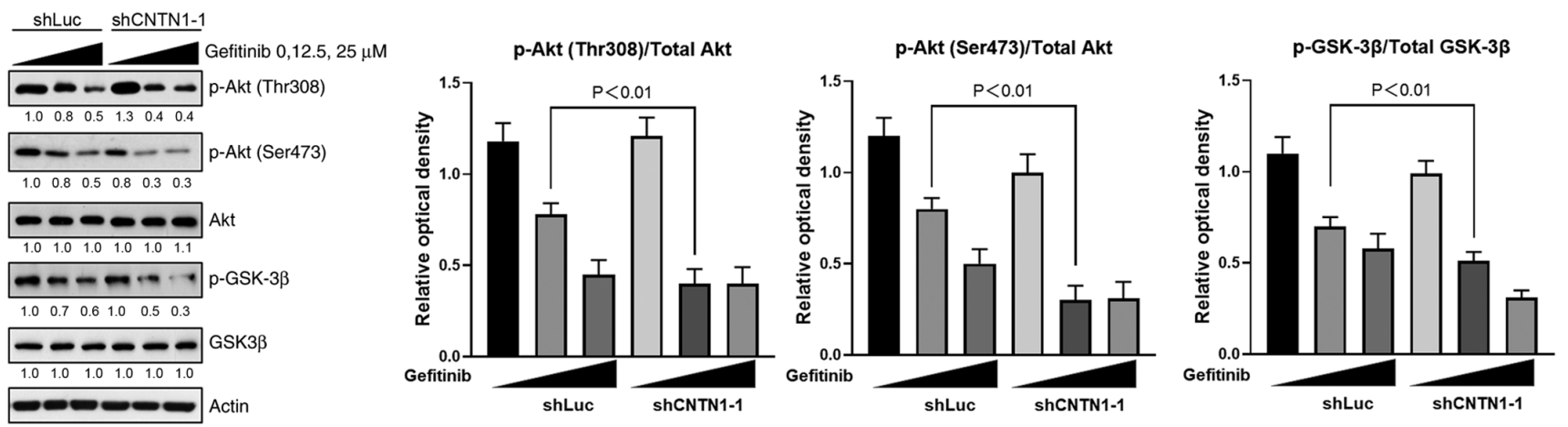

Figure 6. CNTN1 promotes EMT progression and gefitinib resistance by activating the PI3K/Akt signaling pathway in the A549 cells. Stably expressing shLuc or shCNTN1-1 cells were treated with 0,12.5 and $25 \mu \mathrm{M}$ gefitinib for $30 \mathrm{~min}$. The protein expression level of p-Akt (T308), p-Akt (S473), Akt, p-GSK3 $\beta$ and GSK $3 \beta$ was detected using western blot analysis. Actin was used as an internal control. sh, short hairpin; shLuc, control shRNA; $p$, phosphorylated.

the migration and metastasis of cancer cells via EMT, but also promoted drug resistance $(12,15,19,23)$. Therefore, identifying and confirming CNTN1 as a novel therapeutic target associated with EGFR-TKIs resistance is important. The present study aimed to investigate silencing of CNTN1 enhance gefitinib sensitivity by reversing EMT in A549 cell and the mechanism of CNTN1 is described in Fig. 7. The current study found that knockdown of CNTN1 reversed gefitinib resistance and EMT by inactivating the PI3K/Akt signaling pathway and rearranging the cytoskeleton, suggesting that CNTN1 may be a potential target to reverse gefitinib resistance in lung adenocarcinoma A549 cell.

CNTN1, which is located on chromosome 12q11-q12, is a novel member of the contactin sub-group of the immunoglobulin superfamily (29). The expression level and function of CNTN1 has been characterized in the nervous system, and has been associated with the regulation of neurite outgrowth, anchor neural cell adhesion and myelin organization $(30,31)$. Previous studies have demonstrated that CNTN1 played a key role in cancer progression in various types of cancer, including prostate cancer (19), esophageal squamous cell carcinoma (32), oral squamous cell carcinoma (33), hepatocellular carcinoma (14), gastric cancer (13) and LUAD (34). Chen et al (13) reported that CNTN1 protein expression level was increased in patients with primary gastric carcinoma compared with that in adjacent normal gastric mucosa. Similarly, Su et al (35) demonstrated that the mRNA expression level of CNTN1 in the specimens from patients with metastatic LUAD was significantly higher compared with that in samples derived from patients with LUAD without metastasis. In previous studies, CNTN1 promoted cisplatin resistance in cisplatin-resistant lung cancer cells, and knockdown of CNTN1 reversed cisplatin resistance in LUAD $(23,36)$. Consistent with these prior findings, the results from the present study showed that CNTN1 was increased in gefitinib-insensitive and -resistant NSCLC, and knockdown of CNTN1 reversed gefitinib resistance in the A549 cell line. Based on these observations, the present study described the role of CNTN1 in gefitinib resistance and partially investigated the mechanism of CNTN1-mediated EMT.

The EMT process has been associated with migration, invasion and EGFR-TKI resistance in lung cancer. In the clinic, the detection rate of the EMT phenotype was $\sim 14 \%$ in patients with NSCLC and EGFR-TKI resistance (8). Weng et al (37) 


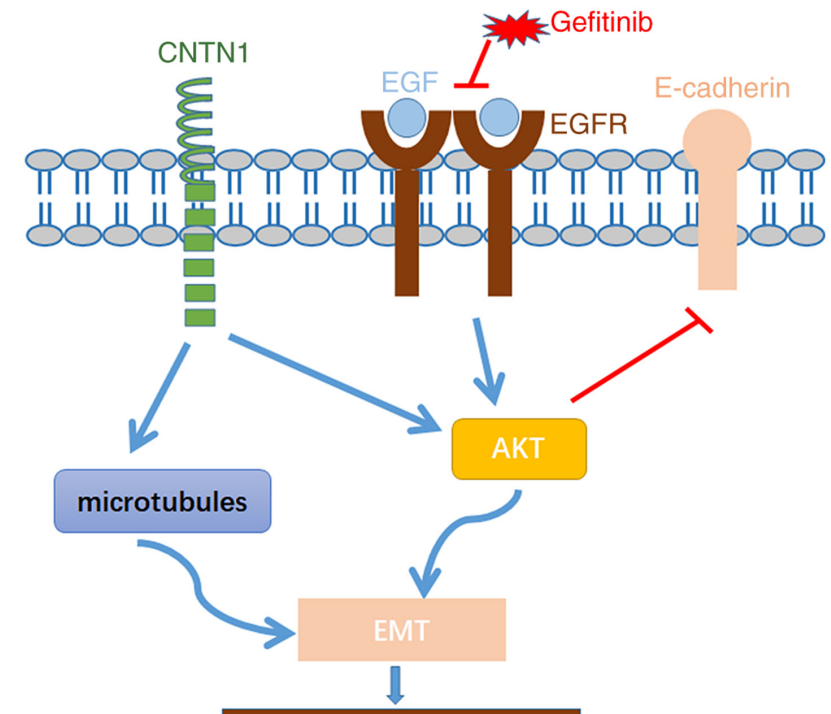

EGFR-TKI resistant

Figure 7. Graphical representation of the mechanism underlying CNTN1-induced EGFR-TKI resistance in non-small cell lung cancer. CNTN1 promotes cell migration and invasion by regulating microtubules to rearrange the cytoskeleton. Furthermore, CNTN1 inhibits E-cadherin expression on the cell membrane by activating Akt, which also results in EMT progression and EGFR-TKI resistance. TKI, tyrosine kinase inhibitor; EMT, epithelial-mesenchymal transition.

reported that gefitinib-resistant cells exhibited an EMT phenotype, with a decrease in E-cadherin protein expression and an increase in vimentin protein expression, which led to resistant cells with enhanced migration and invasion abilities. Furthermore, previous studies showed that CNTN1 rearranged the actin cytoskeleton and focal adhesion structures, and promoted cancer invasion and metastasis $(15,35)$. Overexpression of CNTN1 in the A549 cells promoted EMT progression, and enhanced migration, invasion and cisplatin resistance compared with that in the control cells (23). In the present study, knockdown of CNTN1 reduced cell migration. Further investigation of the mechanism involved indicated that CNTN1 rearranged the cytoskeleton and adhesion structures via the microtubules to affect cell motility. Furthermore, knockdown of CNTN1 upregulated E-cadherin protein expression levels, and downregulated $\mathrm{N}$-cadherin and vimentin protein expression levels. Collectively, CNTN1 overexpression in the A549 cells may contribute to gefitinib resistance and a more aggressive EMT phenotype.

The PI3K/Akt signaling pathway is a vital pathway, that regulates cell proliferation, migration and invasion, as well as drug resistance (24). Activation of the Akt signaling pathway not only inhibited E-cadherin protein expression, for facilitating EMT development, but also promoted gefitinib resistance in NSCLC (23). A previous study showed that Akt phosphorylation (Ser473) was elevated within 5 min after serum stimulation in serum-starved cells and that this was maintained for up to $30 \mathrm{~min}$, which suggests a very fast activation of Akt, which lasts for some time (38). Wang et al (39) showed that Akt phosphorylation (Thr308 and Ser473) increased in serum-starved cells who were subsequently treated with EGF for 5 min or IGF-1 for $30 \mathrm{~min}$. Han et al (40) also demonstrated that
Akt phosphorylation (Thr308 and Ser473) was upregulated within 5 min, after being induced by EGF. Therefore, Akt phosphorylation (Thr308 and Ser473) was detected within 30 min after gefitinib treatment to monitor the rapid Akt pathway activation. However, previous studies reported that Akt phosphorylation was also detectable 6,24 and $48 \mathrm{~h}$ after gefitinib treatment $(41,42)$. There is a limitation on how long inhibition of to the present study Akt activation after gefitinib treatment, as Akt phosphorylation in cells treated with gefitinib for $\geq 1 \mathrm{~h}$ was not investigated.

In the present study, CNTN1 knockdown together with gefitinib treatment significantly inhibited the activation of the PI3K/Akt signaling pathway in the A549 cells. Similar findings have also been reported in lung cancer cell lines. For example, Zhang et al (23) reported that CNTN1 promoted cisplatin resistance in LUAD by inducing EMT progression following activation of the PI3K/Akt signaling pathway. Furthermore, overexpression of CNTN reduced E-cadherin protein expression level to promote lung cancer metastasis, and was associated with the activation of the PI3K/Akt signaling pathway (17). Therefore, CNTN1 could promote gefitinib resistance by activating the PI3K/Akt signaling pathway.

In summary, the present study demonstrated that CNTN1 plays an important role in EGFR-TKI resistance. Knockdown of CNTN1 reversed the EMT phenotype and enhanced gefitinib sensitivity in the A549 cells by inhibiting the activation of the PI3K/Akt signaling pathway. Taken together, these results suggested that CNTN1 may represent a potential therapeutic target for preventing EGFR-TKI resistance in NSCLC.

\section{Acknowledgements}

Not applicable.

\section{Funding}

This study was financially supported by the National Natural Science Foundation of China (grant no. 81902357), and the Science and Technology Research Program of Chongqing University of Arts and Sciences (grant no. 2017RBX10 and R2016BX09).

\section{Availability of data and materials}

The datasets used and/or analyzed in the present study are available from the corresponding author upon reasonable request.

\section{Authors' contributions}

CSH performed experimental work, data analysis, manuscript preparation and editing. JHH assisted with data analysis and acquired funding. DLY assisted with western blotting experiments. CX assisted with bioinformatics analysis. ZGX assisted with data analysis and manuscript preparation. HBT designed the experiments, assisted with data analysis and edited the manuscript. ZZC designed and supervised the experiments, edited the manuscript and acquired funding. CSH and ZZC confirmed the authenticity of all raw data. All authors have read and approved the final manuscript. 


\section{Ethics approval and consent to participate}

Not applicable.

\section{Patient consent for publication}

Not applicable.

\section{Competing interests}

The authors declare that they have no competing interests.

\section{References}

1. Xu Z, Yang Q, Chen X, Zheng L, Zhang L, Yu Y, Chen M, You $\mathrm{Q}$ and Sun J: Clinical associations and prognostic value of site-specific metastases in non-small cell lung cancer: A population-based study. Oncol Lett 17: 5590-5600, 2019.

2. Herbst RS, Morgensztern D and Boshoff C: The biology and management of non-small cell lung cancer. Nature 553: 446-454, 2018.

3. Cho BC, Chewaskulyong B, Lee KH, Dechaphunkul A, Sriuranpong V, Imamura F, Nogami N, Kurata T, Okamoto I, Zhou C, et al: Osimertinib versus standard of care EGFR TKI as first-line treatment in patients with EGFRm advanced NSCLC: FLAURA Asian subset. J Thorac Oncol 14: 99-106, 2019.

4. Han X, Luo R, Wang L, Zhang L, Wang T, Zhao Y, Xiao S, Qiao N, Xu C, Ding L, et al: Potential predictive value of serum targeted metabolites and concurrently mutated genes for EGFR-TKI therapeutic efficacy in lung adenocarcinoma patients with EGFR sensitizing mutations. Am J Cancer Res 10 4266-4286, 2020

5. Nagasaka M,Zhu VW,Lim SM,Greco M,WuF and Ignatius Ou SH: Beyond osimertinib: The development of third-generation EGFR tyrosine kinase inhibitors for advanced EGFR+ NSCLC. J Thorac Oncol: Dec 15, 2020 (Epub ahead of print).

6. Hirsch FR, Scagliotti GV, Mulshine JL, Kwon R, Curran WJ Jr, Wu YL and Paz-Ares L: Lung cancer: Current therapies and new targeted treatments. Lancet 389: 299-311, 2017.

7. Hu C and Tan H: The E3 ubiquitin ligase NEDD4 mediates EGFR-TKI acquired resistance in non-small cell lung cancer. Int J Clin Exp Med 12: 12013-12019, 2019.

8. Sequist LV, Waltman BA, Dias-Santagata D, Digumarthy S, Turke AB, Fidias P, Bergethon K, Shaw AT, Gettinger S, Cosper AK, et al: Genotypic and histological evolution of lung cancers acquiring resistance to EGFR inhibitors. Sci Trans Med 3: 75ra26, 2011.

9. Yue J, Lv D, Wang C, Li L, Zhao Q, Chen H and Xu L: Epigenetic silencing of miR-483-3p promotes acquired gefitinib resistance and EMT in EGFR-mutant NSCLC by targeting integrin $\beta 3$. Oncogene 37: 4300-4312, 2018.

10. Yang X, Peng Y, Jiang X, Lu X, Duan W, Zhang S, Dai N, Shan J, Feng Y, Li X, et al: The regulatory role of APE1 in epithelial-to-mesenchymal transition and in determining EGFR-TKI responsiveness in non-small-cell lung cancer. Cancer Med 7: 4406-4419, 2018.

11. Lou W, Chen Y, Zhu KY, Deng H, Wu T and Wang J: Polyphyllin I overcomes EMT-associated resistance to erlotinib in lung cancer cells via IL-6/STAT3 pathway inhibition. Biol Pharm Bull 40: 1306-1313, 2017

12. Wang B, Yang X, Zhao T, Du H, Wang T, Zhong S, Yang B and $\mathrm{Li} \mathrm{H}$ : Upregulation of contactin-1 expression promotes prostate cancer progression. Oncol Lett 19: 1611-1618, 2020.

13. Chen DH, Yu JW, Wu JG, Wang SL and Jiang BJ: Significances of contactin-1 expression in human gastric cancer and knockdown of contactin-1 expression inhibits invasion and metastasis of MKN45 gastric cancer cells. J Cancer Res Clin Oncol 141: 2109-2120, 2015

14. Li GY, Huang M, Pan TT and Jia WD: Expression and prognostic significance of contactin 1 in human hepatocellular carcinoma. Onco Targets Ther 9: 387-394, 2016.

15. Chen N, He S, Geng J, Song ZJ, Han PH, Qin J, Zhao Z, Song YC Wang HX and Dang CX: Overexpression of contactin 1 promotes growth, migration and invasion in Hs578T breast cancer cells. BMC Cell Biol 19: 5, 2018
16. Shi K, Xu D, Yang C, Wang L, Pan W, Zheng C and Fan L: Contactin 1 as a potential biomarker promotes cell proliferation and invasion in thyroid cancer. Int J Clin Exp Pathol 8 : 12473-12481, 2015

17. Yan J, Wong N, Hung C, Chen WX and Tang D: Contactin-1 reduces E-cadherin expression via activating AKT in lung cancer. PLoS One 8: e65463, 2013.

18. Wu HM, Cao W, Ye D, Ren GX, Wu YN and Guo W: Contactin 1 (CNTN1) expression associates with regional lymph node metastasis and is a novel predictor of prognosis in patients with oral squamous cell carcinoma. Mol Med Rep 6: 265-270, 2012.

19. Yan J, Ojo D, Kapoor A, Lin X, Pinthus JH, Aziz T, Bismar TA, Wei F, Wong N, De Melo J et al: Neural cell adhesion protein CNTN1 promotes the metastatic progression of prostate cancer. Cancer Res 76: 1603-1614, 2016.

20. Coldren CD, Helfrich BA, Witta SE, Sugita M, Lapadat R, Zeng C, Barón A, Franklin WA, Hirsch FR, Geraci MW and Bunn PA Jr: Baseline gene expression predicts sensitivity to gefitinib in non-small cell lung cancer cell lines. Mol Cancer Res 4 521-528, 2006

21. Terai H, Soejima K, Yasuda H, Nakayama S, Hamamoto J, Arai D, Ishioka K, Ohgino K, Ikemura S, Sato T, et al: Activation of the FGF2-FGFR1 autocrine pathway: A novel mechanism of acquired resistance to gefitinib in NSCLC. Mol Cancer Res 11: 759-767, 2013.

22. Gyorffy B, Surowiak P, Budczies J and Lanczky A: Online survival analysis software to assess the prognostic value of biomarkers using transcriptomic data in non-small-cell lung cancer. PLoS One 8: e82241, 2013.

23. Zhang R, Sun S, Ji F, Liu C, Lin H, Xie L, Yang H, Tang W, Zhou Y, Xu J and Li P: CNTN-1 enhances chemoresistance in human lung adenocarcinoma through induction of epithelial-mesenchymal transition by targeting the PI3K/Akt pathway. Cell Physiol Biochem 43: 465-480, 2017.

24. Song M, Bode AM, Dong Z and Lee MH: AKT as a therapeutic target for cancer. Cancer Res 79: 1019-1031, 2019.

25. Liu Q, Yu S, Zhao W, Qin S, Chu Q and Wu K: EGFR-TKIs resistance via EGFR-independent signaling pathways. Mol Cancer 17: 53, 2018

26. Maemondo M, Inoue A, Kobayashi K, Sugawara S, Oizumi S, Isobe H, Gemma A, Harada M, Yoshizawa H, Kinoshita I, et al: Gefitinib or chemotherapy for non-small-cell lung cancer with mutated EGFR. N Engl J Med 362: 2380-2388, 2010.

27. Lei Y, Guo W, Chen B, Chen L, Gong J and Li W: Tumorreleased lncRNA H19 promotes gefitinib resistance via packaging into exosomes in nonsmall cell lung cancer. Oncol Rep 40: 3438-3446, 2018.

28. Cho JH, You YM, Yeom YI, Lee DC, Kim BK, Won M, Cho BC, Kang M, Park S, Yang SJ, et al: RNF25 promotes gefitinib resistance in EGFR-mutant NSCLC cells by inducing NF-kB-mediated ERK reactivation. Cell Death Dis 9: 587, 2018.

29. Berglund EO and Ranscht B: Molecular cloning and in situ localization of the human contactin gene (CNTN1) on chromosome 12q11-q12. Genomics 21: 571-582, 1994.

30. Haenisch C, Diekmann H, Klinger M, Gennarini G, Kuwada JY and Stuermer CA: The neuronal growth and regeneration associated Cntn1 (F3/F11/Contactin) gene is duplicated in fish: Expression during development and retinal axon regeneration. Mol Cell Neurosci 28: 361-374, 2005.

31. Mohebiany AN, Harroch S and Bouyain S: New insights into the roles of the contactin cell adhesion molecules in neural development. Adv Neurobiol 8: 165-194, 2014.

32. Liu P, Chen S, Wu W, Liu B, Shen W, Wang F, He X and Zhang S: Contactin-1 (CNTN-1) overexpression is correlated with advanced clinical stage and lymph node metastasis in oesophageal squamous cell carcinomas. Jap J Clin Oncol 42: 612-618, 2012.

33. Shigetomi S, Imanishi Y, Shibata K, Sakai N, Sakamoto K, Fujii R, Habu N, Otsuka K, Sato Y, Watanabe Y, et al: VEGF-C/Flt-4 axis in tumor cells contributes to the progression of oral squamous cell carcinoma via upregulating VEGF-C itself and contactin- 1 in an autocrine manner. Am J Cancer Res 8: 2046-2063, 2018.

34. Su JL, Yang PC, Shih JY, Yang CY, Wei LH, Hsieh CY, Chou CH, Jeng YM, Wang MY, Chang KJ, et al: The VEGF-C/Flt-4 axis promotes invasion and metastasis of cancer cells. Cancer Cell 9: 209-223, 2006

35. Su JL, Yang CY, Shih JY, Wei LH, Hsieh CY, Jeng YM, Wang MY, Yang PC and Kuo ML: Knockdown of contactin-1 expression suppresses invasion and metastasis of lung adenocarcinoma. Cancer Res 66: 2553-2561, 2006. 
36. Zhang R, Yao W, Qian P, Li Y, Jiang C, Ao Z, Qian G, Wang C, $\mathrm{Wu} \mathrm{G}$ and Li J: Increased sensitivity of human lung adenocarcinoma cells to cisplatin associated with downregulated contactin-1. Biomed Pharmacother 71: 172-184, 2015.

37. Weng CH, Chen LY, Lin YC, Shih JY, Lin YC, Tseng RY, Chiu AC, Yeh YH, Liu C, Lin YT, et al: Epithelial-mesenchymal transition (EMT) beyond EGFR mutations per se is a common mechanism for acquired resistance to EGFR TKI. Oncogene 38: 455-468, 2019.

38. Abkhezr M, Keramati AR, Ostad SN, Davoodi J and Ghahremani MH: The time course of Akt and ERK activation on XIAP expression in HEK 293 cell line. Mol Biol Rep 37: 2037-2042, 2010.

39. Wang G, Long J, Gao Y, Zhang W, Han F, Xu C, Sun L, Yang SC, Lan J, Hou Z, et al: SETDB1-mediated methylation of Akt promotes its K63-linked ubiquitination and activation leading to tumorigenesis. Nat Cell Biol 21: 214-225, 2019.

40. Han F, Li CF, Cai Z, Zhang X, Jin G, Zhang WN, Xu C, Wang CY, Morrow J, Zhang S, et al: The critical role of AMPK in driving Akt activation under stress, tumorigenesis and drug resistance. Nat Commun 9: 4728, 2018.
41. Wang Y, Zhang W, Wen L, Yang H, Wen M, Yun Y, Zhao L, Zhu X, Tian L, Luo E, et al: FOXM1 confers resistance to gefitinib in lung adenocarcinoma via a MET/AKT-dependent positive feedback loop. Oncotarget 7: 59245-59259, 2016.

42. Zhang J, Qu Z, Yao H, Sun L, Harata-Lee Y, Cui J, Aung TN, Liu X, You R, Wang W, et al: An effective drug sensitizing agent increases gefitinib treatment by down regulating PI3K/Akt/mTOR pathway and up regulating autophagy in non-small cell lung cancer. Biomed Pharmacother 118: 109169, 2019.

(c) (3) $\Theta$ This work is licensed under a Creative Commons Attribution-NonCommercial-NoDerivatives 4.0 International (CC BY-NC-ND 4.0) License. 